\title{
Research on Semantic of Updatable Distributed Logic and Its Application in Access Control
}

\author{
Li Ma ${ }^{\mathrm{a}, 1}$, Peng Leng ${ }^{\mathrm{b}}$, Yong Zhong, ${ }^{\mathrm{a}, *}$, Wenyin Yang ${ }^{\mathrm{a}}$ \\ ${ }^{a}$ School of Electronic and Information Engineering, Foshan University, Foshan, China. \\ ${ }^{b}$ Department of Information Engineering, Wuhan Business University, Wuhan, China.
}

\begin{abstract}
The paper presents a distributed logic UD-Datalog whose advantage lies that it extends U-Datalog to distributed environment but still keeps the logic semantic and evaluation method of U-Datalog. The logic presented a new approach to define update in distributed environment based on non-immediate update semantics which distinguishes the language from other distributed datalog. The language is pure declarative and allows us to use top-down and equivalent bottom-up computational evaluation so the already developed techniques for Datalog evaluation can be reused. Firstly, the paper elaborates the syntax and semantic of the logic. Secondly, the evaluation method of the logic is explained. Finally, an application example of the logic in access control of network is discussed which shows the application and expressiveness of the logic.
\end{abstract}

Keywords: Distributed Logic; updatable distributed datalog; access control; UD-Datalog

\section{Introduction}

The emergence of Web 2.0 and social networks have attracted a large amount of users to regularly connect, interact and share information with each other, such as twitter, Facebook, LinkedIn, YouTube. However, the massive data and distributed environment of social networks make traditional access control models difficult to implement $[1,2,3,4]$. So in recent years, logic programming has been proposed as an attractive foundation for distributed programming

\footnotetext{
* Correspondence to Yong Zhong: Electronic and Information Engineering School, Foshan University, Foshan, China. Email: zhongyong@fosu.edu.cn

Email addresses: molly_917@163.com (Li Ma), lp521@sina.com (Peng Leng), zhongyong@fosu.edu.cn (Yong Zhong), cswyyang@163.com (Wenyin Yang)

${ }^{1}$ This work was supported by grants from the National Science Foundation of Guangdong Province No.2015A030313638, National Spark Plan Project under Grant No. 2014GA780031, and Provincial Major Project of Guangdong under Grant 2014WZDXM036, and the Foundation for Distinguished Young Talents in Higher Education of Guangdong in China under Funding Support Number 2015KQNCX179.
} 
based on work in declarative networking [5]. And there has been optimism that declarative languages grounded in Datalog can provide a clean foundation for distributed programming, which makes a hit for research of distributed computation using such languages $[6,7,8,9,10,11,12,13]$. However, in these papers, the semantics is operational and based on a distribution of the program before the execution.

In view of issues with this model, Joseph presents a new model DEDALUS based on an explicit time constructor [14]. But Galland found the semantics of negation together with the use of time in that model rather unnatural. In particular, time is used as an abstract logical notion to control execution steps and the future may have influence on the past. As a consequence, it is difficult to understand what applications are doing as well as to prove results on their language. Galland presents WebdamLog language for the solutions which still is unnatural for its difficult for distinguishing deductive or active rules or update semantics [15]. And the semantics is still operational.

Especially for updatable state, many deductive database systems admit procedural semantics to deal with updates using an assignment primitive. DEDALUS retain a purely logical interpretation by admitting temporal extensions into their syntax and interpreting assignment or update as a composite operation across time steps rather than as a primitive [16]. Many distributed logic languages use hybrid declarative/imperative languages such as Overlog [17] or Boom [18][19] and other $[20,21,7,14,22]$ often clouded our understanding of the "correct" execution of single-node programs that performed state updates due to the combination of Datalog and imperative constructs.

The paper presents a distributed logic UD-Datalog whose contributions can be summarized as follows:

- The logic extends U-Datalog to distributed environment but still keep$\mathrm{s}$ the logic semantic and evaluation method of U-Datalog in centralized environment so a universal logic can be kept.

- The logic presented a new approach to define update in distributed environment based on non-immediate update semantics which distinguishes the language from other distributed datalog, since an immediate update is the main feature of current distributed datalog.

- The language is pure declarative and allows us to use top-down and equivalent bottom-up computational evaluation so the already developed techniques for Datalog evaluation can be reused, which leads a evaluation convenience and advantages comparing to current hybrid declarative/imperative logic languages in distributed environment.

The remainder of the paper will be organized as follows. Section 2 provides preliminaries covering the concepts of U-Datalog and distributed DU-Datalog. Section 3 gives details of our proposed model, which is the logic semantic. Section 4 illuminates the universal evaluation algorithm of UD-Datalog program with an implementation example. An application example of access control is 
showed in Section 5 and a contrastive analysis is described in in Section 6. The paper is concluded in Section 7.

\section{Preliminary}

\subsection{Overview of U-Datalog}

U-Datalog is a type of updatable Datalog programming language, in which predicate atoms consist of both updated atoms representing Insert and Remove, expressed as $\pm p\left(t_{1}, t_{2}, \ldots, t_{n}\right)$. U-Datalog language can be considered a special use case of using Constraint Logic Programming (CLP), by which updating atoms $\pm p\left(t_{1}, t_{2}, \ldots, t_{n}\right)$ as the constraints of the Datalog rules. Except updatable atoms, other Datalog atoms are Query Atoms (QA) phase. Two implementation phases of U-Datalog include Marking and Update. The marking phase completes the variable binding of the transactions and obtains the update sets. The update phase implements the update sets.

Moreover, transactions in U-Datalog follow the rule in Eq. (1) without carrying headers.

$$
U_{1}, \ldots, U_{k}, L_{1}, \ldots, L_{m}
$$

In Eq. (1), $U_{i}(0 \leqslant i \leqslant k)$ is a random update atom. $L_{i}(0 \leqslant i \leqslant m)$ is an arbitrary literal. For any transaction $T$, we define that a $T$ 's query atom set is the query transaction of $T$. The update set obtained during the marking phase is T's update transaction. Additionally, a pure Datalog program can be considered a special case of U-Datalog.

\subsection{Distributed DU-Datalog Rules}

\subsubsection{Domain}

A domain is the value range of a predicate variable, and we call a discrete finite domain a finite domain. A predicate can be showed as $p\left(x_{1}: t_{1}, \ldots, x_{n}\right.$ : $t n)$, where we call $x_{1}, \ldots, x_{n}$ the terms of corresponding domain and $t_{i}(1 \leqslant$ $i \leqslant n)$ are the types of corresponding terms. If domain of type $t_{i}(1 \leqslant i \leqslant n)$ is a finite domain, we call $x_{i}(1 \leqslant i \leqslant n)$ a finite term. For simplicity, we omit predicate type and show a predicate as $p\left(x_{1}, \ldots, x_{n}\right)$.

\subsubsection{Predicate Types and Entity (Location) Suffix}

We assume the last term of each predicate $p\left(x_{1}, \ldots, x_{n}\right)$ represents the entity of the predicate. For a intuitive view, we express the predicate $p\left(x_{1}, \ldots, x_{n-1}\right) @ x_{n}$ which means the predicate $p\left(x_{1}, \ldots, x_{n-1}\right)$ on the entity $x_{n}$. We use $\operatorname{Loc}\left(p\left(x_{1}, \ldots, x_{n}\right)\right)$ to represent entity $x_{n}$. Similar to Webdamlog [1], an entity is an object having storage and data processing capabilities, which can be either a physical network node or a virtual entity, such as an account in social networks. 


\subsubsection{Predicate and Atoms}

We define a constant a term and a variable a term. Let $p\left(x_{1}, \ldots, x_{n-1}\right) @ x_{n}$ be a random predicate symbol, with the terms $x_{1}, \ldots, x_{n-1}$ in its corresponding domain and $x_{n}$ is the entity suffix. We call $p\left(x_{1}, \ldots, x_{n-1}\right) @ x_{n}$ or $\pm p\left(x_{1}, \ldots, x_{n-1}\right) @ x_{n}$ an atom formula or atom for short. Predicates includes two types: extensional predicates shown as $P_{\text {ext }}$ and intensional predicates shown as $P_{\text {int }}$. Atoms include two types:

1. Update atoms. $\pm p\left(x_{1}, \ldots, x_{n-1}\right) @ x_{n}$ that represent the insertion or deletion of corresponding extensional predicates are called update atoms.

2. Query atoms. The atoms except update atoms are called query atoms. We call atom $p\left(x_{1}, \ldots, x_{n-1}\right) @ x_{n}$ or its negation $-p\left(x_{1}, \ldots, x_{n-1}\right) @ x_{n}$ as a literal. Negations are constrained to extensional predicates, that is $p\left(x_{1}, \ldots, x_{n-1}\right) @ x_{n}$ in body of a rule must be extensional predicates. The constraint is the simplest situation of applying negative atoms but it is enough to satisfy with our requirement. For length of the paper, we do not discuss the negative atoms or negative literals further here.

\subsubsection{Programs and Transactions}

Extensional Database (EDB) consists of ground atoms that represent database status. And Intensional Database (IDB) consists of the rule below:

$$
h @ e \leftarrow u_{1} @ e_{1}, \ldots, u_{k} @ e_{k}, p_{k+1} @ e_{k+1}, \ldots, p_{m} @ e_{m}
$$

In Eq. (2), $h$ is a random query atom, $p_{i}(k+1 \leqslant i \leqslant m)$ is a random query literal, and $u_{i}(1 \leqslant j \leqslant k)$ is a random update atom.

Transaction (query) $T$ is a kind of rule without head that has the form:

$$
u_{1} @ e_{1}, \ldots, u_{k} @ e_{k}, p_{k+1} @ e_{k+1}, \ldots, p_{m} @ e_{m}
$$

Where $p_{i}(k+1 \leqslant i \leqslant m)$ is a query literal, and $u_{i}(1 \leqslant j \leqslant k)$ is a update atom. We call the set of query literal in $T$ as query transaction of $T$. And call the update set an update transaction brought by execution of transaction $T$. The paper uses ? - $\left(u_{1} @ e_{1}, \ldots, u_{k} @ e_{k}, p_{k+1} @ e_{k+1}, \ldots, p_{m} @ e_{m}\right)$ to represent a transaction or query. A shortened form representing a transaction with the same suffix is given as follows:

$$
\left\{T_{1}\right\} @ e_{1}, \ldots,\left\{T_{m}\right\} @ e_{m}
$$

If all the entities in a transaction $T$ are $e$, we call the transaction a single transaction on entity e. In Eq. (4), all $T_{i}(1 \leqslant j \leqslant m)$ can be looked on as a single transaction, the same as traditional transaction. Sometimes, we omit the entity suffix in a single transaction. In addition, we require the rules in a program are safe, which means all variables occurring in the head also appear in the body and any variables in the body must appear in a sub-goal that is not a negative or the variables come from a finite domain. The safe rules guarantee all variables are restricted, which prevent that an unrestricted variable brings rules or facts that cannot be controlled by the database. 


\section{Proposed Model and Concepts}

In this section, we describe our proposed approach that is Distributed DUDatalog Semantic (DDS).

\subsection{Regularity of the Rule}

Definition 1 defines the regular deduction rule.

Definition 1. Regular Deduction Rule: the deduction rule for any entity $i$ follows the rule given in Eq. (5).

$$
h @ e \leftarrow u_{1} @ e_{1}, \ldots, u_{k} @ e_{k}, p_{k+1} @ e_{k+1}, \ldots, p_{m} @ e_{m}
$$

where $h$ is a random query atom, $p_{i}(k+1 \leqslant i \leqslant m)$ is a random query literal, $u_{i}(1 \leqslant j \leqslant k)$ is a random update atom. The deduction rule is regular if the suffix at the rule header has $e=i$.

Definition 2. Regular Rule System: Assume that entity e's deduction rule set is regular, then we call this entity has a regular rule system. We call the network system $N$ 's rule system is regular when any entity's rule system in the network system follows the regularity.

The regularity is to ensure the result of the deduction rule is the local internal predicate. The following examples in the papers all assume that entity's deduction rule is regular as the following example of Alice's laptop (Alice-laptop):

$$
\begin{aligned}
& \text { friend }(\text { Alice }, x) @ \text { Alice }- \text { laptop } \leftarrow \text { addresslist }(x, y) @ \text { Alice }-i \text { Phone } \\
& \text { friend(Alice, } x) \leftarrow \text { direct_relation(Alice, } x \text {, "friend")@facebook_account, } \\
& \text { depth(Alice, } x \text {, "friend", } m \text { )@facebook_account }, m \leqslant 2 @ \text { facebook_account }
\end{aligned}
$$

Rule (6) describes that Alice's laptop cognizes the user inAlice's iPhone's contact as Alice's friend. Rule (7) shows that Alice's laptop cognizes the user whose relation depth is not bigger than 2 in Alice's facebook account as Alice's friend. In rule (7), the rule header omits the local entity suffix. We simplify the entity predicate in the rule in order to have a clear expression as follows.

$$
\begin{aligned}
& \text { friend(Alice, } x) \leftarrow\{\text { direct_relation(Alice, } x, \text { "friend"), } \\
& \operatorname{depth}(\text { Alice, } x, \text { "friend", } m), m \leqslant 2\} @ \text { facebook_account }
\end{aligned}
$$

Therefore, the regular rule on entity $e$ can be represented as the following rule:

$$
h \leftarrow\{T\},\{T 1\} @ e_{1}, \ldots,\left\{T_{n}\right\} @ e_{n}
$$

In Rule 9, $\operatorname{Loc}(h)=e, \operatorname{Loc}(T)=e, \operatorname{Loc}\left(T_{i}\right)=e_{i}(1 \leqslant i \leqslant n) . T$ is the transaction on entity $e . T_{i}(1 \leqslant i \leqslant n)$ is the transaction on the corresponding entity $e_{i}$. 


\subsection{Semantic Implementation}

Definition 3. Mapping Set on Entity of Entity Update Set: Assume that the update set $\tilde{U}=\left\{u_{1}, u_{2}, \ldots, u_{n}\right\}$. For entity $e$, define that the set formed by all updates from entity $e$ in the update set is the mapping set of the update set $\tilde{U}$ upon entity e, represented as $\lambda(\tilde{U}, e)$.

Definition 4. Consistency of the Entity Update Set: Assume that update set $\tilde{U}=\left\{u_{1}, u_{2}, \ldots, u_{n}\right\}$ and its mapping ensemble set on entity $e$ is $\lambda(\tilde{U}, e)$. Define that the update set $\tilde{U}$ on entity e is of consistency when $\lambda(\tilde{U}, e)$ matches the following rule, represented as ident $(\tilde{U}, e)$ :

$\forall \delta p(\tilde{X}) \in \tilde{u} \Rightarrow ! \exists \gamma p(\tilde{X}) \in \tilde{U} \bigwedge \delta \neq \gamma$

where $\delta, \gamma \in\{+,-\}$

Definition 4 explains that DU-Datalog utilizes a type of weak update. The $\lambda(\tilde{U}, e)$ 's update set is of consistency if $\lambda(\tilde{U}, e)$ does not synchronously contain atoms $+p(\tilde{X})$ and $-p(\tilde{X})$.

Definition 5. Full Consistency of Update Set: Assume that the update set is $\tilde{U}=\left\{u_{1}, u_{2}, \ldots, u_{n}\right\}$. We define that the update set $\tilde{U}$ is fully consistent if any entity e has an ident $(\tilde{U}), e)$ represented as univesal $(\tilde{U})$.

Definition 6. Operational Semantic: Assume that $D B$ is a regular rule set on entity e. We define that the operational semantic $O_{e}(D B)$ of $D B$ on entity e is:

$O_{e}(D B)=\left\{p(\tilde{X}) \leftarrow \tilde{U},\left\{T_{1}\right\} @ e_{1}, \ldots,\left\{T_{n}\right\} @ e_{n} \mid p(\tilde{X}) \leftarrow_{D B}^{\times} \tilde{U},\left\{T_{1}\right\} @ e_{1}, \ldots,\left\{T_{n}\right\} @ e_{n}\right\}$

$\tilde{U}$ is the conjunctive normal form of the update atoms on entity e. $p(\tilde{X})$ is a random atom on entity e except update atom. $p(\tilde{X}) \leftarrow_{D B}^{\times} \tilde{U},\left\{T_{1}\right\} @ e_{1}, \ldots,\left\{T_{n}\right\} @ e_{n}$ means $p(\tilde{X}) \leftarrow \tilde{U},\left\{T_{1}\right\} @ e_{1}, \ldots,\left\{T_{n}\right\} @ e_{n}$ is a derivation from $D B$.

Thus, defining DU-Datalog's Herbrand base $\beta$ on entity $e$ can be formed by the follows: $p(\tilde{X}) \leftarrow \tilde{U},\left\{T_{1}\right\} @ e_{1}, \ldots,\left\{T_{n}\right\} @ e_{n}$, where $\tilde{U}$ is the update atom conjunctive normal form on entity $e, p(\tilde{X})$ is a random atom on entity $e$ except update atom. We define the implementation of the operator below.

Definition 7. Immediate Consequence Operator: Assume that $D B$ is the regular rule set on entity $e$, and $I$ is an interpretation. We define $T_{D B}^{e}$ 's immediate consequence operator is $2^{\beta} \rightarrow 2^{\beta}$.

$$
\begin{aligned}
& T_{D B}^{e}(I)=\left\{p(\tilde{X}) \leftarrow \tilde{u},\left\{T_{1}\right\} @, \ldots,\left\{T_{n}\right\} @ e_{n}\right\} \\
& \exists a \operatorname{renaming} \text { rule: } \\
& p \tilde{X} \leftarrow \tilde{U},\left\{T_{1}\right\} @ e_{1}, \ldots,\left\{T_{n}\right\} @ e_{n}-\in D B \\
& \theta=m g u\left(\left(\left(p_{1}\left(\tilde{Y}_{1}\right), \ldots, p_{m}\left(\tilde{Y}_{m}\right)\right),\left(\left(p_{1}\left(\tilde{Y}_{1}\right), \ldots, p_{m}\left(\tilde{Y}_{m}\right)\right)\right)\right.\right. \\
& \left.\forall i=1, \ldots, m, p_{i}\left(\tilde{X}_{1}\right) \leftarrow \tilde{U}_{i},\left\{T_{i 1}\right\} @ e_{1}, \ldots,\left\{T_{i n}\right\} @ e_{n}\right) \in I \\
& p(\tilde{X})=\theta_{p}(t) \\
& \tilde{U}=\theta\left(\tilde{U}_{0} \cup \tilde{U}_{1} \cup \ldots \cup \tilde{U}_{m}\right) \\
& \operatorname{ident}(\tilde{U}, e) \\
& T_{1}=\theta\left(\tilde{T}_{1}^{\prime} \cup \tilde{T}_{11} \cup \ldots \tilde{T}_{1 m}\right)
\end{aligned}
$$




$$
T_{n}=\theta\left(\tilde{T}_{n}^{\prime} \cup \tilde{T}_{n 1} \cup \ldots \tilde{T}_{n m}\right)
$$

where $\theta$ is the minimum unifier operator, and the function mgu returns a minimum unifier. The update set on entity e is consistent.

Definition 8. Fixpoint Semantic: Assume that $D B$ is the regular rule set on entity e. We define that the fixpoint semantic of $D B$ on entity $e$ is $F i x_{e}(D B)=$ $T_{D B}^{e} \uparrow \omega$, also represented as Fix (e).

Theorem 1. Consistency of Operational Semantic and Fixpoint Semantic: Assume that $D B$ is the regular rule set on entity e, we have $O_{e}(D B)=$ $F_{i x}(D B)$.

Theorem 1 is correct to U-Datalog rule set or Constraint Logic Programming(CLP) on single entity [23]. To entity, if we consider the atom formulae on other entities $\left\{T_{1}\right\} @ e_{1}, \ldots,\left\{T_{n}\right\} @ e_{n}$ a special constraint type, we can use similar method to prove that Theorem 1 is correct. $T_{D B}^{e}$ operator is continuous and monotone increasing and there also exists a least fixpoint, according to Definition 8.

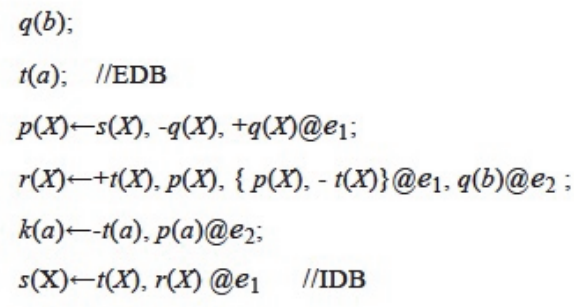

Figure 1: Example of regular DU-Datalog program on entity $e$.

Fig. 1 illustrates an example of regular DU-Datalog program on entity $e$. We can obtain the following results by using direct operator.

1. $T_{0}(D B)=\left\{q(b) ; t(a) ; k(a) \leftarrow-t(a), p(a) @ e_{2}\right\}$

2. $T_{1}(D B)=\left\{s(a) \leftarrow r(a) @ e_{1} ; p(a) \leftarrow-q(a),\{r(a),+q(a)\} @ e_{1}\right\} T 0(D B)$

3. $T_{2}(D B)=\left\{r(a) \leftarrow+t(a),-q(a),\{r(a),+q(a), p(a),-t(a)\} @ e 1, q(b) @ e_{2}\right\} \cup$ $T_{1}(D B)$

4. $T_{3}(D B)=T_{2}(D B)=$ Fixe $_{e}(D B)$

We have $O_{e}(D B)=$ Fixe $_{e}(D B)=T_{3}(D B)=$ Fix $(e)$ based on Theorem 1 .

According to [23], we define $b_{\mid T}=\{(X=t) \in b \mid X$ occurs in $T\}$ if given a set of bindings $b$ and a transaction $T$, and define $\operatorname{eqn}(\theta)=\left\{V_{1}=t_{1}, \ldots, V_{n}=t_{n}\right\}$ if given a substitution $\theta=\left\{V_{1} \leftarrow t_{1}, \ldots, V_{n} \leftarrow t_{n}\right\}$.

Definition 9. Query Update Set and Output Transaction Set: Given the regular rule set $D B$ on entity $e$, as well as its fixpoint Fix $x_{e}(D B)$ and single 
transaction on entity e $T=\left\{\tilde{U}_{0}, p_{1}\left(\tilde{X}_{1}\right), \ldots, p_{m}\left(\tilde{X}_{m}\right)\right\}$. We define $T$ 's update set $\operatorname{Set}_{-} \hat{U}\left(T, F_{i x}(D B)\right)$ in DB under the fixpoint semantic of Fix $(D B)$ as:

$$
\begin{aligned}
& i \leqslant m \\
& \quad \operatorname{Set} \hat{U}\left(T, F i x_{e}(D B)\right)=\left\{\langle b, \tilde{U}\rangle \mid p_{i}\left(\tilde{Y}_{i}\right) \leftarrow \tilde{U}_{i},\left\{T_{i 1}\right\} @ e_{1}, \ldots,\left\{T_{i n}\right\} @ e_{n} \in F i x_{e}(D B), 1 \leqslant\right. \\
& \quad \theta=\operatorname{mgu}\left(\left(\left(p_{1}\left(\tilde{X}_{1}\right), \ldots, p_{m}\left(\tilde{X}_{m}\right)\right),\left(p_{1}\left(\tilde{Y}_{1}\right), \ldots, p_{m}\left(\tilde{T}_{m}\right)\right)\right)\right. \\
& \quad b=\operatorname{eqn}(\theta)_{\mid T} \\
& \left.\quad \tilde{U}=\theta\left(\tilde{U}_{0}, \tilde{U}_{1}, \ldots, \tilde{U}_{n}\right)\right\}
\end{aligned}
$$

We define T's output transaction set $\operatorname{Set}_{-} \hat{T}\left(T, F i x e(D B), e_{i}\right)$ in $D B$ to $e_{i}$ under the fixpoint semantic of Fixe $(D B)$ as:

$$
\begin{aligned}
& \operatorname{Set}_{2} \hat{T}\left(T, F i x_{e}(D B), e_{i}\right)=\left\{<b, T_{i}>\mid\right. \\
& p_{i}\left(\tilde{Y}_{i}\right) \leftarrow \tilde{u}_{i},\left\{T_{i 1}\right\} @ e_{1}, \ldots,\left\{T_{i n}\right\} @ e_{n} \in \operatorname{Fix}_{e}(D B), 1 \leqslant i \leqslant m \\
& \theta=m g u\left(\left(p_{1}\left(\tilde{X}_{1}\right), \ldots, p_{m}\left(\tilde{X}_{m}\right)\right),\left(p_{1}\left(\tilde{Y}_{1}\right), \ldots, p_{m}\left(\tilde{Y}_{m}\right)\right)\right) \\
& b=\operatorname{eqn}(\theta) \mid T \\
& T_{i}=\theta\left(T_{i 1} \cup \cdots \cup T_{i n}\right) \\
& \}
\end{aligned}
$$

Assume that the network system $N=\left\{e_{0}, e_{1}, \ldots, e_{n}\right\}$ and its rule system is regular. $D B_{0}, D B_{1}, \ldots, D B_{n}$ are regular set of the corresponding entities. Assume that transaction $T_{0}^{0}$ is a single transaction activity on entity $e_{0}$. We give the transaction execution diagram in Fig. 2 if the output transaction set is non-empty.

In Fig. 2, we use number 0-n to represent entities $e_{0}, e_{1}, \ldots, e_{n} . T_{i j}^{k}$ represents output transaction, where $k$ refers to the $\mathrm{k} t h$ round output, $i$ represents the entity $e_{i}$ executing the transaction that brings the output transaction, and $j$ represents the entity $e_{j}$ which the output transaction is submitted to. If we merge the output transactions submitted to the same entity $e_{j}$ into a new transaction in every round and the subscript only show the number of the entity $e_{j}$, we get a new transaction that merges the output transactions submitted to entity $e_{j}$ in $\mathrm{k} t h$ round. We call the new transaction $T_{j}^{k}$ single output transaction to entity $e_{j}$ in kth round. For example, as shown in Fig. 2, the second round output transaction $T_{24}^{2}$ and $T_{44}^{2}$ can be merged to single output transaction $T_{4}^{2}$ to entity $e_{4}$ in second round. If we look on as a start node and every single output transaction as a node, then we can get a Directed Transaction Execution Graph (DTEG), also Transaction Execution Graph (TEG) as Fig. 3.

We call the transaction hierarchy of T's Directed Transaction Execution Graph a full hierarchy of $T$.

Definition 10. Hierarchy: The hierarchy is a quaternion set $\left(M_{i}, M_{a}, M, \leqslant\right)$, where

1. $M_{i}, M_{a}$, and $M$ are mutually disjoint sets, which means $M_{i} \cap M_{a}=$ $\emptyset \wedge M_{i} \cap M=\emptyset \wedge M_{a} \cap M=\emptyset$.

$2 . \leqslant$ refers to partial ordering relations for the set $\left(M_{i} \cup M_{a} \cup M\right)$. For any $m\left(m \in M_{i}\right)$ that is minimum in the set $\left(M_{i} \cup M_{a} \cup M\right)$, it needs to match the condition that there is no element smaller than $m$ at the hierarchy, as $n \leqslant m \Rightarrow n=m$. For any $m \in M_{a}$ is maximum in the set $\left(M_{i} \cup M_{a} \cup M\right)$, 


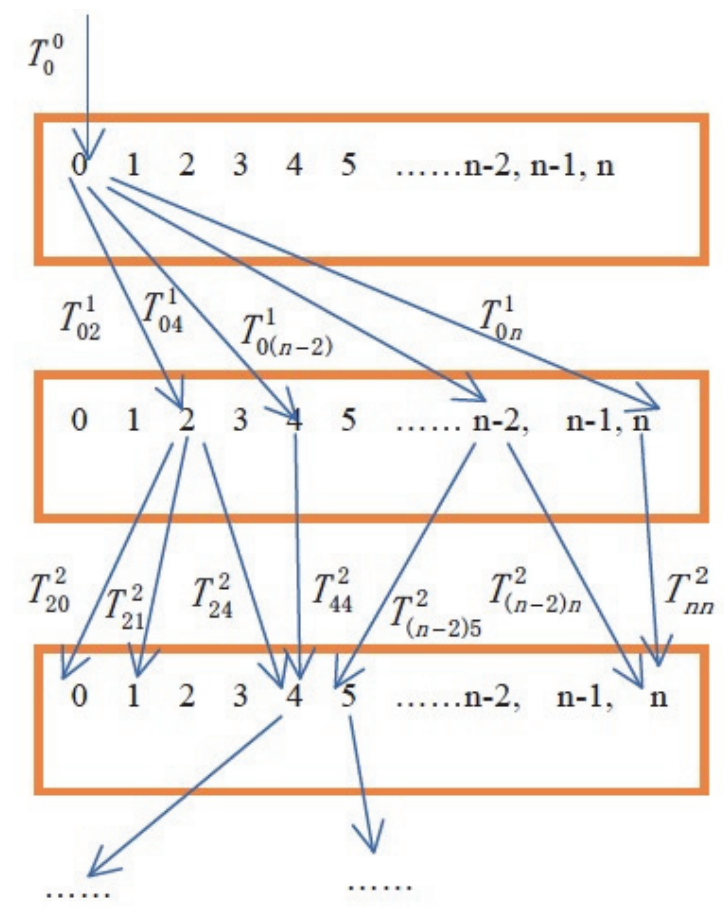

Figure 2: Diagram of the transaction execution for $T_{0}^{0}$.

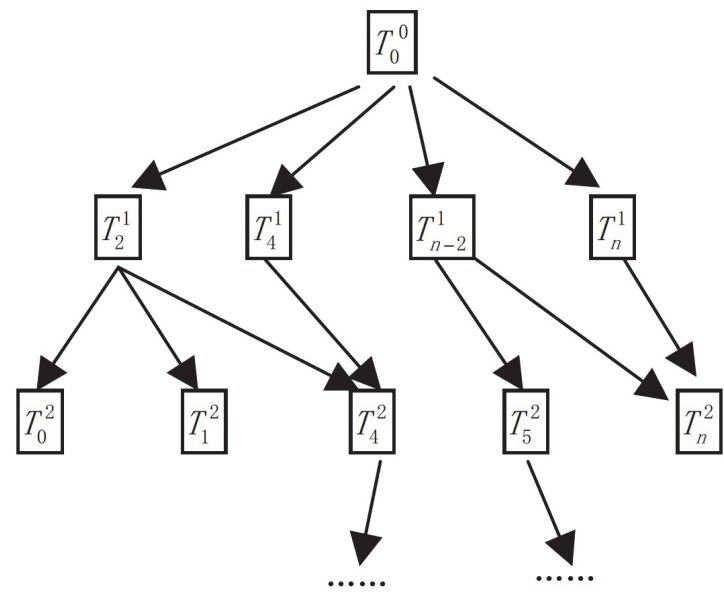

Figure 3: Directed transaction implementation graph for $T_{0}^{0}$.

it needs to match the condition that there is no element bigger than $m$ at the hierarchy, as $m \leqslant n \Rightarrow n=m$. 
Definition 11. Transaction Hierarchy: Assume that $N=\left\{e_{0}, e_{1}, \ldots, e_{n}\right\}$ is a regular network system. Transaction Hierarchy $(\mathrm{TH})$ is a quaternion set $\left(T_{i}, T_{a}, T, \leqslant_{T}\right)$, where $T_{i}, T_{a}$, and $T(k>0)$ are the identifier set. We use $T_{i}^{k}$ to present the kth output transaction to the entity $e_{j}$, and $\leqslant_{T}$ refers to the partial ordering relation, where follows three conditions below:

1. Any $T_{i}^{k} \in T_{i} \Rightarrow k=0$.

2. Any $T_{i}^{k} \in T \Rightarrow k>0$.

3. Any $T_{i}^{k}, T_{j}^{m} \in T_{i} \cup T, T_{i}^{k} \leqslant_{T} T_{j}^{m} \Rightarrow m=k+1 \wedge \operatorname{Set} \hat{T}\left(T, F i x_{e i}\left(D B_{e i j}\right), e_{j}\right) \neq$ $\emptyset$.

The condition 3 states that the requirement of $T_{i}^{k} \leqslant_{T} T_{j}^{m}$ is that the transaction $T_{i}^{k}$ has an output transaction to $e_{j}$ at the (k-1)th round. We call the transaction hierarchy of T's Directed Transaction Execution Graph a full hierarchy of $T$.

Definition 12. Ancestral Relationship of Transaction Hierarchy: $A s$ sume that $N=\left\{e_{0}, e_{1}, \ldots, e_{n}\right\}$ is a regular network system. TH is a full hierarchy of the transaction $T_{0}^{0}$. We define that any partial ordering relations $\leqslant_{P}$ following the conditions below is the ancestral relationship of the transaction hierarchy.

1. For any $T_{i}$ and $T_{j}$ on hierarchy, $T_{i} \leqslant_{T} T_{j} \Rightarrow T_{i} \leqslant_{P} T_{j}$

2. For any $T_{i}, T_{j}$, and $T_{k}$ on hierarchy, $T_{i} \leqslant_{T} T_{j} \wedge T_{j} \leqslant_{P} T_{k} \Rightarrow T_{i} \leqslant_{P} T_{k}$

Condition 1 represents the hierarchy relation is an ancestral relationship. Condition 2 represents the transaction is of ancestral relationship if it has a hierarchy relationship with its ancestor. For $T_{i} \leqslant_{P} T_{j}$, we call $T_{i}$ is the ancestral node of $T_{j}$.

Definition 13. Transaction Loop Lattic (TLL): Assume that $N=\left\{e_{0}, e_{1}, \ldots, e_{n}\right\}$ is a regular network system. TH is a full hierarchy of the transaction $T_{0}^{0}$. Assume that there exists an hierarchy $T L L=\left(T_{i}, T_{a}, T, \leqslant_{T}\right)$. We call $T L L$ is the transaction loop lattice on TH if TLL follows the relations below:

1. $\# T_{i}=1 \wedge \# T_{a}=1$, the cardinal number of the transaction sets $T_{i}$ and $T_{a}$ is 1 , then there is only one maximum value and there is only one minimum value, $T_{i}^{k} \in T_{i} \Rightarrow k=0$.

2. $T_{i}^{k} \in T_{i} \wedge T_{j}^{m} \in T_{a} \Rightarrow m>k \wedge T_{i}^{k} \subseteq T_{j}^{m}$

3. $T_{i}^{k} \in T_{i} \wedge T_{j}^{m} \in T_{a} \Rightarrow ! \exists T_{j}^{k} i \neq j \wedge T_{j}^{k} \leqslant_{P} T_{j}^{m}$

Condition 1 represents the transaction loop lattice only has one minimum value and one maximum value. The transaction having the minimum value is the subset of the transaction of the maximum value in condition 2. Condition 3 represents that there is no transaction $T_{j}^{k}$ that is at the same round with the minimum value transaction $T_{i}^{k}$. This transaction is also the ancestral node of the maximum value transaction. The minimum value transaction is the only ancestral node at this round. 


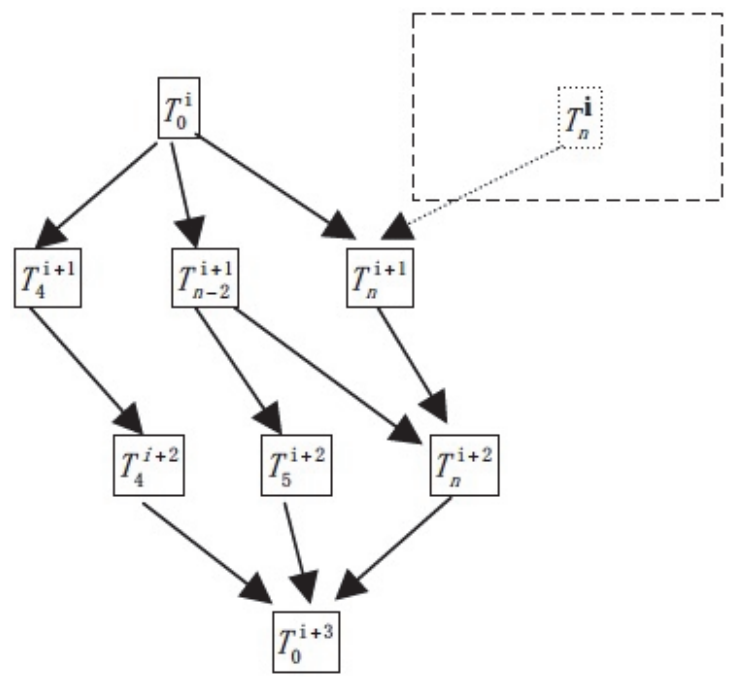

Figure 4: Example of transaction loop lattice.

Fig. 4 gives an example of the transaction loop lattice, where $T_{0}^{i} \subseteq T_{0}^{i+3}$. The shown example will not be a transaction loop lattice if there exists an ancestral node shown in the broken lines.

Theorem 2. Transaction Terminability: The directed transaction implementation graph that does not contain transaction loop lattice is terminable.

Proof. Assume that $N=\left\{e_{0}, e_{1}, \ldots, e_{n}\right\}$ is a regular network system. The directed execution graph of transaction $T_{0}^{0}$ is given in Fig. 3.

First, we need to prove that the directed transaction execution graph including transaction loop lattice is non-terminable. As shown in Fig. 4, $T_{0}^{i} \subseteq T_{0}^{i+3}$, sending $T_{0}^{i+3}$ to transaction $e_{0}$ can result in multiple existences of the same lattice at different rounds, if $T_{0}^{i}=T_{0}^{i+3}$. It make the transaction loop lattice repeated occurrence in the directed transaction execution graph that makes the transaction non-terminable. For $T_{0}^{i} \subset T_{0}^{i+3}$, we can consider $T_{0}^{i+3}$ a set $\left(T_{0}^{i} \cup\right.$ another transaction), which can result in repeated existences of the same lattice.

If directed execution graph does not include any transaction loop lattice, the rules and facts in any entity $e$ are finite, and the current interpretation $I$ is finite, so the number of transactions that can be submitted to entity $e$ is also finite, which means the number of different nodes in Fig. 3 is finite and the number of different structure of transaction execution graph is finite. If a transaction execution graph is not terminable, after some rounds, some nodes and their structure must be repeated to form a transaction loop lattice. Thus, if a transaction execution graph does not include transaction loop lattice, it must be terminable. Proved. 


\section{Algorithms}

The assessment algorithm of DU-Datalog program consists of mark and update phases. The purpose of mark phase is to calculate the update set of the transactions. Update phase is to update the network entities.

Define that $N=\left\{e_{0}, e_{1}, \ldots, e_{n}\right\}$ is a regular network system. TH is a full hierarchy of the transaction $T_{0}^{0}$. TG is transaction $T_{0}^{0}$ 's directed execution graph. TH's transactions of $i$ th rounds are stored in the set $\mathrm{TH}[\mathrm{i}]$.

\subsection{Transaction Loop Lattice Detection Algorithm}

This algorithm is designed to detect whether there exist transaction loop lattices in the full hierarchy.

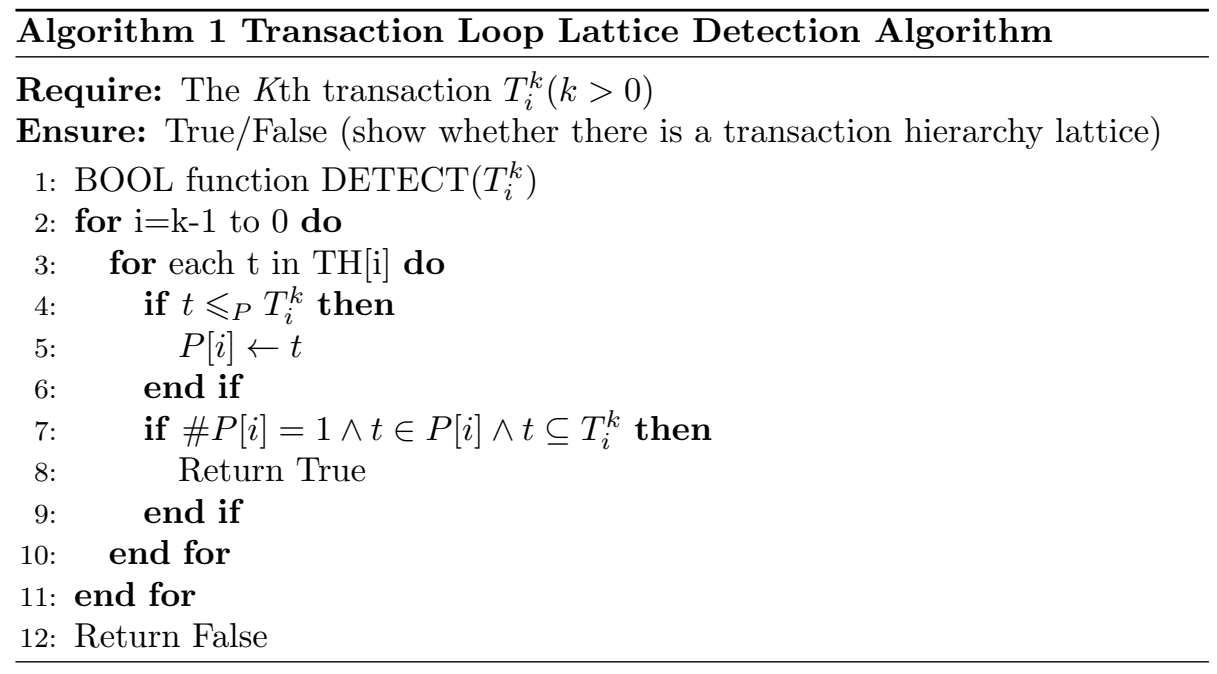

\subsection{Mark Phase Algorithm}

The algorithm for the make phase can use not only a bottom-up algorithm to get the fixpoint like seminavie or its variant which induces the update set, but also some top-down optimization algorithms like magic set or WF (constraint memoing) to get update set directly. In our algorithm, we use a bottom-top method and design a breadth-first search method to search all updates. An FALSE will be returned once a transaction loop lattice is found at the full hierarchy of transaction $T_{0}^{0}$ or transaction cannot be satisfied by the program. Otherwise, the updates brought by successful execution of transaction will be collected to array $U[0, \ldots, n]$ of update set.

\subsection{Update Phase Algorithm}

The purpose of the update phase algorithm is to implement $U[0, \ldots, n]$ caused by the transaction $T_{0}^{0}$. DU-Datalog update semantic is a type of weak 


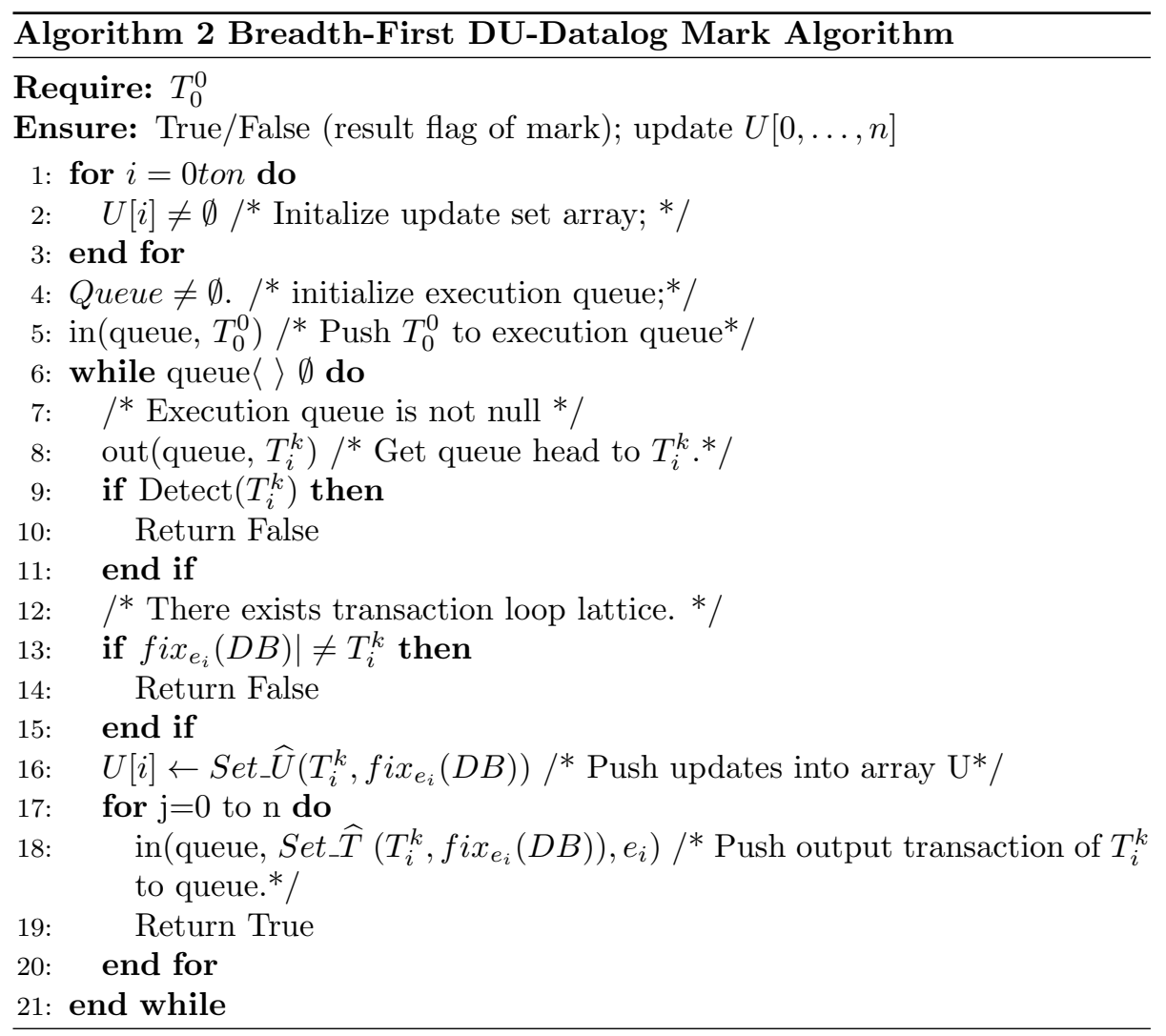

update [23]. In a weak update environment, there is no need to know the status of EDB. The update will not be consistent only when $+p(\tilde{t})$ and $-p(\tilde{t})$ are concurrently in the update transaction set $\mathrm{U}[\mathrm{i}], 0 \leqslant i \leqslant n$.

Definition 14. Update Incorporation Function: Given update set $U$ on entity $e$ and external database $E D B_{0}, E D B_{1}, \ldots, E D B_{n}(n \geqslant 0)$ of the entity, we define the update incorporation function:

incorp $(U, E D B)=\cup_{i}$ in $(U, E D B i)$ where $1 \leqslant i \leqslant n, E D B=E D B 0 \cup E D B 1 \cup$ $\cdots \cup E D B_{n}$

To current external database status $E D B_{i}$, update $\langle b,+p(\tilde{a})>\in U$, where $p(\tilde{a})$ is ground atom(fact), then function in is:

$\operatorname{in}\left(U, E D B_{i}\right)=E D B_{i} \cup p()$

To current external database status $E D B_{i}$ update $<b,-p(\tilde{a})>\in U$, where $p(\tilde{a})$ is ground atom(fact), then function in is:

in $\left(U, E D B_{i}\right)=E D B_{i} \backslash p(\tilde{a})$

To current external database status $E D B_{i}$, update $<b, \pm p(\tilde{a})>\in U$, where $p(\tilde{)}$ includes unground variables. So execution of update operation cannot be decided, only a null operation can be executed, then function in is: in $\left(U, E D B_{i}\right)=E D B_{i}$ To current external database status $E D B_{i}$, updates are inconsistent, then the 
update operation is failed, the transaction will not be submitted.

The algorithm of update phase detects the consistency of update array firstly, then calls update incorporation function to update the EDB. Updates are executed by a two-phase commit. The steps are described in Algorithm 3.

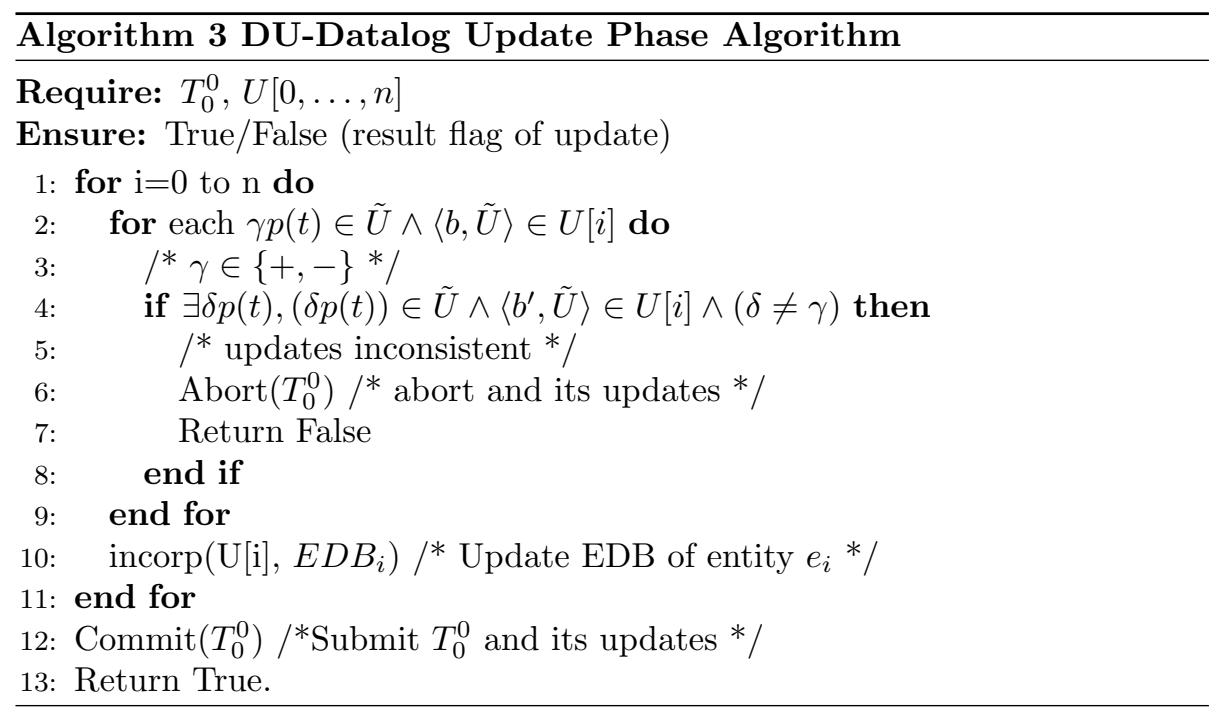

\subsection{Example for Algorithm Process}

Given entity $e_{0}, e_{1}$, and $e_{2}$ as Fig. 5 , if transaction $T_{0}^{0}=(p(x), r(x), k(a))$ : 


\begin{tabular}{|ll|}
\hline$q(b) ;$ & \\
$t(a) ; / / \mathrm{EDB}$ & Entity $e_{0}$ \\
$p(X) \leftarrow s(X),-q(X),+q(X) @ e_{1} ;$ & \\
$r(X) \leftarrow+t(X), p(X),\{p(X),-t(X)\} @ e_{1}, q(b) @ e_{2} ;$ \\
$k(a) \leftarrow+t(a), p(a) @ e_{2} ;$ \\
$s(X) \leftarrow t(X), r(X) @ e_{1} \quad / / \mathrm{IDB}$ & \\
\hline$q(c) ;$ & Entity $e_{1}$ \\
$q(d) ;$ & \\
$t(b) ; \quad / / \mathrm{EDB}$ & \\
$p(X) \leftarrow q(X),+q(X) ;$ & \\
$r(X) \leftarrow-t(X), p(X), p(X) @ e_{2} ;$ & \\
$k(X) \leftarrow t(X), q(X) @ e_{2} ; \quad / / \mathrm{IDB}$ & \\
\hline$q(a) ;$ & \\
$q(b)$ & \\
$t(b) ; \quad / / \mathrm{EDB}$ & \\
$p(X) \leftarrow q(X) @ e_{1}, q(X) ;$ & \\
$r(X) \leftarrow+t(X), p(X),+p(X) @ e_{1} ;$ & \\
$k(X) \leftarrow-t(X), p(X) @ e_{1}, q(X) ; \quad / / \mathrm{IDB}$ & \\
\hline
\end{tabular}

Figure 5: Entities $e_{0}, e_{1}$, and $e_{2}$. 
1. Compute fixpoint of $e_{0}, e_{1}$, and $e_{2}$ :

$$
\begin{aligned}
& \text { Fix }\left(e_{0}\right)=\{q(b) ; t(a) ; \\
& k(a) \leftarrow+t(a), p(a) @ e_{2} ; \\
& s(a) \leftarrow r(a) @ e_{1} ; \\
& p(a) \leftarrow-q(a),\{r(a),+q(a)\} @ e_{1} \\
& \left.r(a) \leftarrow+t(a),\{r(a),+q(a), p(a),-t(a)\} @ e_{1}, q(b) @ e_{2}\right\}
\end{aligned}
$$

$F i x\left(e_{1}\right)=\{q(b) ; q(d) ; t(b) ;$

$$
\begin{aligned}
& p(a) \leftarrow+q(a), p(d) \leftarrow+q(d) ; \\
& r(a) \leftarrow-t(a),+q(a), p(a) @ e_{2} \\
& r(d) \leftarrow-t(d),+q(d), p(d) @ e_{2} \\
& \left.k(b) \leftarrow q(b) @ e_{2} ;\right\}
\end{aligned}
$$

$F i x\left(e_{2}\right)=\{q(a) ; q(b) ; t(b) ;$

$$
\begin{aligned}
& p(a) \leftarrow q(a) @ e_{1} ; \\
& p(b) \leftarrow q(b) @ e_{1} ; \\
& r(a) \leftarrow+t(a), q(a) @ e_{0},+p(a) @ e_{1} ; \\
& r(b) \leftarrow+t(b), q(b) @ e_{0},+p(b) @ e_{1} ; \\
& k(a) \leftarrow-t(a), q(a) @ e_{2} ; \\
& \left.k(b) \leftarrow-t(b), q(b) @ e_{2} ;\right\}
\end{aligned}
$$

2. According to Algorithm 2, the first round update set and output transaction set is as following:

$$
\begin{aligned}
& U[0]=\{-q(a),+t(a)\} \\
& U[1]=\{+q(a),-t(a)\} \\
& U[2]=\{\} \\
& T_{0}^{1}=\{\} \\
& T_{1}^{1}=\{r(a), p(a)\} \\
& T_{2}^{1}=\{q(b)\}
\end{aligned}
$$

3. The second round update set and output transaction set is as following:

$$
\begin{aligned}
& U[0]=\{-q(a),+t(a)\} \\
& U[1]=\{+q(a),-t(a),\} \\
& U[2]=\{\} \\
& T_{0}^{2}=\{\} \\
& T_{1}^{2}=\{p(a)\} \\
& T_{2}^{2}=\{\}
\end{aligned}
$$


4. The third round update set and output transaction set is as following:

$$
\begin{aligned}
& U[0]=\{-q(a),+t(a)\} \\
& U[1]=\{+q(a),-t(a),\} \\
& U[2]=\{\} \\
& T_{0}^{2}=\{\} \\
& T_{1}^{2}=\{\} \\
& T_{2}^{2}=\{\}
\end{aligned}
$$

5. After entity update, the result is as Fig. 6. The output transaction set is empty, which means the mark phase is end. Then the update phase start according to the Algorithm 3.

\begin{tabular}{|l|}
$q(b) ;$ \\
$t(a) ; \quad / / \mathrm{EDB}$ \\
$p(X) \leftarrow s(X),-q(X),+q(X) @ e_{1} ;$ \\
$r(X) \leftarrow+t(X), p(X),\{p(X),-t(X)\} @ e_{1}, q(b) @ e_{2} ;$ \\
$k(a) \leftarrow+t(a), p(a) @ e_{2} ;$ \\
$s(X) \leftarrow t(X), r(X) @ e_{1} \quad / / \mathrm{IDB}$ \\
\hline$q(a) ;$ \\
$q(c) ;$ \\
$q(d) ;$ \\
$t(b) ; \quad / / \mathrm{EDB}$ \\
$p(X) \leftarrow q(X),+q(X) ;$ \\
$r(X) \leftarrow-t(X), p(X), p(X) @ e_{2} ;$ \\
$k(X) \leftarrow t(X), q(X) @ e_{2} ; \quad / / \mathrm{IDB}$ \\
\hline$q(a) ;$ \\
$q(b)$ \\
$t(b) ; \quad / / \mathrm{EDB}$ \\
$p(X) \leftarrow q(X) @ e_{1}, q(X) ;$ \\
$r(X) \leftarrow+t(X), p(X),+p(X) @ e_{1} ;$ \\
$k(X) \leftarrow-t(X), p(X) @ e_{1}, q(X) ; \quad / / \mathrm{IDB}$ \\
\hline
\end{tabular}

Figure 6: Entities $e_{0}, e_{1}$, and $e_{2}$ after updates.

\section{Motivational Example}

Here we show the application of the UD-logic in access control of a network system. Alice can login in the network from her account and she have a laptop 
and a cell phone. How the login can be controlled and the fee is computed. We show how the access control policies can be set by the UD-logic rules. There are are five entities in network system $N$. The virtual entity Alice's account (AliceAccount) and four real physical entities, including Alice's laptop (Alice-laptop), Alice's cell phone (Alice-phone), the login server (Login-server), and a fee count server (Fee-server).

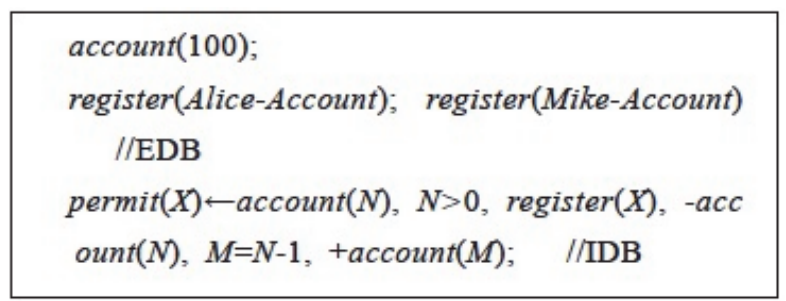

Figure 7: The regular rule of login-server.

Fig. 7 represents the regular rule of login-server. Account(100) means the number of the total permitted login is 100. Register is the predicate of account login. $I D B$ shows that login account is allowed when the number of the permitted login is greater than 0 and this account was logged in. When the account is logged in, the number of the permitted login minus 1.

$$
\begin{array}{ll}
\operatorname{price}(5) ; \operatorname{deposit}(\text { Alice-Account, 50) } & / / \mathrm{EDB} \\
\operatorname{consume}(X) \leftarrow \operatorname{price}(N), \quad \operatorname{deposit}(X, \quad M), \quad M>N, \\
L=M-N,-\operatorname{deposit}(X, M),+\operatorname{deposit}(X, L) ; & / / \mathrm{IDB}
\end{array}
$$

Figure 8: The regular rule of fee-server.

Fig. 8 represents the regularity rule of fee-server. Price(5) shows that the cost fee is 5 for each login. Deposit(Alice-Account, 50) means that Alice has 50 deposit. $I D B$ rule says that it allows $X$ to login when the deposit amount is greater than the cost of each login. The cost of login will be deducted at each time of login.

Fig. 9 represents the regular rule of Alice-account. EDB rule states that Alice-account has two devices, which are Alice-laptop and Alice-phone Rule 1 of $I D B$ says that an entity must obtain the permission from login server and have enough money to pay the fee before he can login the system. Rule 2 of IDB says that an entity $X$ must have been registered and can checkin the network before he can login the network, where self represents the entity itself.

Finally, both Alice-laptop and Alice-phone follow the regular rule below: $\operatorname{login}($ Self $) \leftarrow \operatorname{login}($ Self $) @$ Alice - Account. This rule describes that Alice's laptop or phone can be logged in the system only when they can be logged in 


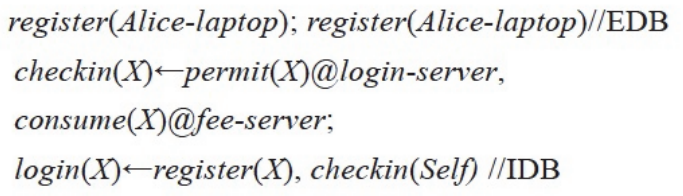

Figure 9: The regularity rule of Alice-account.

the Alice's account.

\section{Discussions}

Comparing to current distributed logic, DU-Datalog presented a new approach to define update in distributed environment based on non-immediate update semantics which distinguishes the language from other distributed datalog. And the language is pure declarative and allows us to use top-down and equivalent bottom-up computational evaluation so the already developed techniques for U-Datalog evaluation can be reused as the analysis in the following Table 1. The logic extends U-Datalog to distributed environment but still keeps the logic semantic and evaluation method of U-Datalog. In Webdamlog language peers exchange includes not only facts but also rules which brings some complexity also some expressiveness to the language.

Table 1: An Contrastive Analysis of Distributed Logic

\begin{tabular}{|c|c|c|c|c|}
\hline & Overlog [17] & WebdamLog [15] & Dedalus [16] & DU-Datalog \\
\hline Declarative/Imperative & Hybrid & Hybrid & Hybrid & Declarative \\
\hline Update & Immediate & Immediate & Immediate & Non-immediate \\
\hline Updatable State & Primitive & Primitive & Composite operation & Constraint logic \\
\hline Logic Semantic & Changed & Changed & Changed & Unchanged \\
\hline Peers Exchange & Facts & Facts & Facts and rules & Facts \\
\hline
\end{tabular}

\section{Conclusions}

The paper presents a distributed logic UD-Datalog whose extends U-Datalog to a distributed environment. The syntax, semantic and evaluation method of the logic is discussed. The logic defines update in distributed environment based on non-immediate update semantics and is pure declarative which distinguishes the language from other distributed datalog. By the example, we can know the logic will be a powerful tool for access control model. In our future work, we will incorporate time factor and trigger mechanism into the logic, which will make the logic a more powerful expressiveness. And the logic will be used in access control model for online social network. 


\section{References}

[1] L. Ma, L. Tao, K. Gai, and Y. Zhong. A novel social network access control model using logical authorization language in cloud computing. Concurrency and Computation: Practice and Experience, PP(99):1, 2016.

[2] M. Qiu, M. Zhong, J. Li, K. Gai, and Z. Zong. Phase-change memory optimization for green cloud with genetic algorithm. IEEE Transactions on Computers, 64(12):3528 - 3540, 2015.

[3] H. Yin and K. Gai. An empirical study on preprocessing high-dimensional class-imbalanced data for classification. In The IEEE International Symposium on Big Data Security on Cloud, pages 1314-1319, New York, USA, 2015 .

[4] M. Qiu, K. Gai, B. Thuraisingham, L. Tao, and H. Zhao. Proactive usercentric secure data scheme using attribute-based semantic access controls for mobile clouds in financial industry. Future Generation Computer Systems, PP:1, 2016.

[5] Chen Chen, Lay Kuan Loh, Limin Jia, Wenchao Zhou, and Boon Thau Loo. Automated verification of safety properties of declarative networking programs. In Proceedings of the 17th International Symposium on Principles and Practice of Declarative Programming, PPDP '15, pages 79-90, New York, NY, USA, 2015. ACM.

[6] T. Ameloot, F. Neven, and J. Bussche. Relational transducers for declarative networking. Journal of the ACM (JACM), 60(2):15, 2013.

[7] J. Navarro and A. Rybalchenko. Operational semantics for declarative networking. In International Symposium on Practical Aspects of Declarative Languages, pages 76-90. Springer, 2009.

[8] J. Pérez, A. Rybalchenko, and A. Singh. Cardinality abstraction for declarative networking applications. In International Conference on Computer Aided Verification, pages 584-598. Springer, 2009.

[9] L. Ma, L. Tao, Y. Zhong, and K. Gai. Rulesn: Research and application of social network access control model. In IEEE International Conference on Intelligent Data and Security, pages 418-423, New York, USA, 2016. IEEE.

[10] S. Abiteboul, M. Bienvenu, A. Galland, and E. Antoine. A rule-based language for web data management. In Proceedings of the thirtieth ACM SIGMOD-SIGACT-SIGART symposium on Principles of database systems, pages 293-304. ACM, 2011.

[11] K. Gai, M. Qiu, M. Chen, and H. Zhao. Security-aware efficient data transmission for ITS in mobile heterogeneous cloud computing. ACM Transactions on Embedded Computing Systems, PP(99):1, 2009. 
[12] K. Gai, M. Qiu, H. Zhao, and J. Xiong. Privacy-aware adaptive data encryption strategy of big data in cloud computing. In 2016 IEEE 3rd International Conference on Cyber Security and Cloud Computing, pages 273-278. IEEE, 2016.

[13] Y. Li, K. Gai, L. Qiu, M. Qiu, and H. Zhao. Intelligent cryptography approach for secure distributed big data storage in cloud computing. Information Sciences, $\mathrm{PP}(99): 1,2016$.

[14] J. Hellerstein. The declarative imperative: experiences and conjectures in distributed logic. ACM SIGMOD Record, 39(1):5-19, 2010.

[15] A. Galland. Distributed data management with access control social networks and data of the web. Nature Communications, 5:4864-4864, 2011.

[16] Peter Alvaro, William R. Marczak, Neil Conway, Joseph M. Hellerstein, David Maier, and Russell Sears. Dedalus: Datalog in time and space. In International Conference on Datalog Reloaded, pages 262-281, 2010.

[17] B. Loo and W. Zhuo. Declarative networking. Communication of the ACM, 52(11):87-95, 2009.

[18] P. Alvaro, T. Condie, N. Conway, K. Elmeleegy, J. Hellerstein, and R. Sears. Boom analytics: exploring data-centric, declarative programming for the cloud. In Proceedings of the 5th European conference on Computer systems, pages 223-236. ACM, 2010.

[19] P. Alvaro, T. Condie, N. Conway, K. Elmeleegy, J. Hellerstein, and R. Sears. BOOM: data-centric programming in the datacenter. EECS Department, University of California, Berkeley, Tech. Rep. UCB/EECS-2009-113, 2009.

[20] P. Alvaro, T. Condie, and N. Conway. I do declare: consensus in a logic language. In The Fifth International Workshop on Networking Meets Databases, pages 25-30, 2010.

[21] S. Abiteboul, P. Bourhis, and V. Vianu. A formal study of collaborative access control in distributed datalog. In ICDT 2016-19th International Conference on Database Theory, 2016.

[22] V. Zaychik Moffitt, J. Stoyanovich, S. Abiteboul, and G. Miklau. Collaborative access control in webdamlog. In Proceedings of the 2015 ACM SIGMOD International Conference on Management of Data, pages 197211. ACM, 2015.

[23] E. Bertino, B. Catania, V. Gervasi, and A. Raffaetà. Active-u-datalog: Integrating active rules in a logical update language. In Workshop on (Trans) Actions and Change in Logic Programming and Deductive Databases, pages 107-133. Springer, 1997. 